\title{
Comparison of a Convected Helmholtz and Euler Model for Impedance Eduction in Flow
}

\author{
Willie R. Watson* and Michael G. Jones ${ }^{\dagger}$ \\ NASA Langley Research Center, Hampton, Virginia 23681-2199, USA
}

\begin{abstract}
Impedances educed from a well-tested convected Helmholtz model are compared to that of a recently developed linearized Euler model using two ceramic test liners under the assumed conditions of uniform flow and a plane wave source. The convected Helmholtz model is restricted to uniform mean flow whereas the linearized Euler model can account for the effects of the shear layer. Test data to educe the impedance is acquired from measurements obtained in the NASA Langley Research Center Grazing Incidence Tube for mean flow Mach numbers ranging from 0.0 to 0.5 and source frequencies ranging from $0.5 \mathrm{kHz}$ to $3.0 \mathrm{kHz}$. The unknown impedance of the liner is educed by judiciously choosing the impedance via an optimization method to match the measured acoustic pressure on the wall opposite the test liner. Results are presented on four spatial grids using three different optimization methods (contour deformation, Davidon-Fletcher Powell, and the Genetic Algorithm). All three optimization methods converge to the same impedance when used with the same model and to nearly identical impedances when used on different models. An anomaly was observed only at $0.5 \mathrm{kHz}$ for high mean flow speeds. This anomaly is likely due to the use of measured data in a flow regime where shear layer effects are important but are neglected in the math models. Consistency between the impedances educed using the two models provides confidence that the linearized Euler model is ready for application to more realistic flows, such as those containing shear layers.
\end{abstract}

\section{Nomenclature}

$[A],\{B\} \quad=$ finite element system matrix and vector of source effects, Pa

$a, d=$ diameter and depth of the parallel cylindrical channels composing the test liner, $\mathrm{m}$

$c, k=$ ambient sound speed, $\mathrm{m} / \mathrm{s}$, and free space wave number, $1 / \mathrm{m}$

$F(\zeta) \quad=$ wall error function, $\mathrm{Pa}^{2}$

$f, \omega=$ source frequency, $\mathrm{Hz}$, and angular frequency, $\mathrm{s}^{-1}$

$H, W, L \quad=$ height, width, and length of duct, $\mathrm{m}$

$i \quad=\sqrt{-1}$, unit imaginary number

$L_{1}, L_{2} \quad=$ location of the leading and trailing edge of wall lining, $\mathrm{m}$

$M(x, y, z) \quad=$ mean flow Mach number

$M_{0}(x), M_{C} \quad$ = fully developed mean flow Mach number and centerline Mach number

$M_{\text {ave }} \quad=$ average mean flow Mach number over three axial stations

$N$, nwall $=$ order of $[A]$ and number of lower wall measurement points

$P, T, \rho \quad=$ mean flow pressure, $\mathrm{Pa}$, temperature, $\mathrm{K}$, and density, $\mathrm{kg} / \mathrm{m}^{3}$

$p \quad=$ frequency domain acoustic pressure, $\mathrm{Pa}$

$\theta, \chi \quad=$ dimensionless acoustic resistance and reactance normalized by $\rho_{0} c_{0}$

$t \quad=$ time, $\mathrm{s}$

$u, v \quad=$ frequency domain axial and transverse acoustic particle velocity, $\mathrm{m} / \mathrm{s}$

$x, y, z=$ transverse, spanwise, and axial coordinate, $\mathrm{m}$

$\zeta=\theta+i \chi$, dimensionless acoustic impedance normalized by $\rho_{0} c_{0}$

$\{\Phi\} \quad=$ vector of unknown node pressures, $\mathrm{Pa}$

* Senior Research Scientist, Computational AeroSciences Branch, Senior Member of AIAA.

${ }^{\dagger}$ Senior Research Scientist, Structral Acoustics Branch, Senior Member of AIAA. 


$$
\begin{aligned}
& \text { Subscripts: } \\
& 0 \quad=\text { mean flow quantity } \\
& \text { exit } \quad=\text { exit plane quanity } \\
& \text { FEM, Meas = finite element computed and measured acoustic pressure } \\
& \text { I } \quad \text { = index of summation } \\
& \text { ref } \quad=\text { standard day condition at } 295 \mathrm{~K} \\
& s \quad=\text { source plane } \\
& \text { c } \quad=\text { centerline }
\end{aligned}
$$

Superscripts:

$$
\text { * }=\text { complex conjugate }
$$

\section{Introduction}

$\mathrm{E}$

FFICIENT duct liners for broadband acoustic noise suppression remain critical to the development of environmentally acceptable commercial aircraft. Previous experiments ${ }^{1}$ have shown that significant changes in duct liner impedance can occur as a result of grazing flow. Because of the need to better understand this phenomenon, NASA Langley Research Center (LaRC) has invested a considerable effort into the development of convected Helmholtz equation $(\mathrm{CHE})^{2-4}$ and linearized Euler (LEE) ${ }^{5}$ models to educe impedance in grazing flow. Although the CHE model is restricted to uniform flow, the LEE model can account for the effects of the shear layer. In both models, the test section in the LaRC $51 \times 51 \mathrm{~mm}$ Grazing Incidence Tube (GIT) is used to acquire the acoustic and mean flow data needed to educe the impedance of the test liner. Impedances educed using the CHE model have been extensively reported $^{2-4}$ and compare favorably to normal incidence impedance tube results at Mach zero. Inconsistencies in the impedance educed from the CHE model have been observed at the lowest frequency (i.e., $0.5 \mathrm{kHz}$ ) at high mean flow speeds. To date, no satisfactory explanation for this anomaly has been given.

In 2002, results from the CHE model were compared to results of methods used in various U.S. aeroacoustics laboratories. ${ }^{6}$ Impedance data comparisons acquired from this multi-laboratory study suggested the need to incorporate the shear layer and 3-D aeroacoustic effects into the impedance eduction methodologies. Subsequently in 2003, a 95-microphone window and improved mean flow suction devices were installed in the GIT $^{7}$ to obtain aeroacoustic and improved mean flow measurements for impedance eduction in 3-D sound fields with shear layers. In 2005, a model was developed ${ }^{4}$ for educing liner impedance using aeroacoustic and mean flow data obtained from the modified GIT.

Although the LEE model ${ }^{5}$ can account for the shear layer, it has yet to be extensively validated even in uniform mean flow. One would expect that impedances educed using the CHE and LEE models would be identical for the same frequency, source condition, mean flow field, and liner. In addition, several time domain computational aeroacoustics (CAA) models that are being developed to model the effects of duct liners have tended to use GIT data for validation purposes. ${ }^{8-11}$ Although these CAA models assume an anechoic (nonreflecting) termination, the suitability of this assumption within the context of the GIT has not been determined. For example, if the CAA and GIT results show significant differences in the frequency range where the GIT termination is not anechoic, it would be difficult to determine whether such discrepancies were due to termination effects or other reasons. Educing the impedance with the measured and nonreflecting termination and demonstrating that the educed impedance is minimally affected could determine the suitability of using an anechoic boundary condition with GIT data.

This paper provides a description of one of the first applications of the LEE model to data acquired in the modified GIT. GIT measurements obtained for ceramic tubular test liners are used to provide a "sanity check" on the LEE model by showing that it reproduces the educed impedances of the more extensively tested CHE model in uniform flow. The current research also provides an opportunity for the authors to showcase some recently developed tools that significantly enhance the efficiency and accuracy of the impedance eduction methodology. Among these are a parallel, sparse, equation solver (that significantly reduces the wall clock time and computer memory requirements) and the Genetic algorithm (that adds increased robustness to the technique and renders it extremely attractive for massively parallel computing architectures). These new tools are used to extensively probe the acoustic solution in the vicinity of the duct wall and explain the anomaly in educed impedance at $0.5 \mathrm{kHz}$ for high Mach number flows. To determine whether an anechoic termination is a suitable assumption for validating CAA codes using GIT data, the impedance educed using the measured termination is compared to that using an anechoic termination. 


\section{Statement of Problem}

FIGURE 1 is a schematic of the modified LaRC GIT and Cartesian coordinate system used in this study. The source F and exit planes are located $203 \mathrm{~mm}$ upstream and downstream, respectively, of the leading and trailing edges of the test liner. The $406 \mathrm{~mm}$ long test liner $\left(L_{2}-L_{1}=406 \mathrm{~mm}\right)$ is located on the upper wall of the GIT. The two sidewalls and bottom wall of the duct are rigid and the test liner is assumed uniform and locally reacting with an unknown dimensionless liner impedance, $\zeta$. The cross section of the GIT is $51 \mathrm{~mm}$ in width and $51 \mathrm{~mm}$ in height. Although the duct exit plane was designed to be anechoic, measurements show that reflections occur at sufficiently high mean flow Mach numbers. To account for these reflections, the exit plane impedance is measured and used as input to the impedance eduction method. It is assumed that the acoustic pressure field along the wall opposite the test liner is known from the wall mounted microphones (provided, for example, by the 95 -microphone window ${ }^{7}$ ). Finally there is a steady, nonuniform, mean flow field that flows subsonically from left to right with mean flow Mach number, $M_{0}$, as shown in Fig. 1. The problem at hand is to determine the unknown impedance, $\zeta$, of the test liner in the presence of the flowing fluid.

\section{Duct Propagation Models}

$\mathbf{I}^{\mathrm{N}}$ this section a brief summary of two duct propagation models (the linearized Euler equations and convected 1 Helmholtz equation models) that are solved to educe the liner impedance is presented. The physical geometry (uniform cross section and test specimen, with rigid sidewalls) and restricted operational frequency range $(f=0.5-3.0$ $\mathrm{kHz}$ ) of the GIT, allow only two-dimensional acoustic disturbances to propagate. This situation is exploited by restricting the analyses to two space dimensions. Only a summary of the relevant differential equations and boundary conditions are presented. More detailed derivations may be found in any standard aeroacoustics text.

\section{A. Linearized Euler Equations (LEE) Model}

The governing differential equations for the 2-D LEE model are described by the 2-D linearized Euler equations

$$
\begin{gathered}
i k p+M_{0} \frac{\partial p}{\partial z}+\rho_{0} c_{0}\left(\frac{\partial u}{\partial z}+\frac{\partial v}{\partial x}\right)=0 \\
i k u+M_{0} \frac{\partial u}{\partial z}+\frac{1}{\rho_{0} c_{0}} \frac{\partial p}{\partial z}+\frac{d M_{0}}{d x} v=0 \\
i k v+M_{0} \frac{\partial v}{\partial z}+\frac{1}{\rho_{0} c_{0}} \frac{\partial p}{\partial x}=0
\end{gathered}
$$

where the acoustic disturbance is assumed to take place isentropically in an ideal gas and a time dependence of the form $e^{i \omega t}$ has been assumed. Implicit in the above derivation is that the mean flow field is fully developed (i.e., $M_{0}=M_{0}(x)$, $\rho_{0}=$ constant,$P_{0}=$ constant,$T_{0}=$ constant $)$.

The proper forms of the boundary conditions have been well established. The wall impedance boundary condition for a locally reacting impenetrable surface of arbitrary shape and mean flow has been presented by Myers ${ }^{12}$

$$
\rho_{0} c_{0} v=\left(1+\frac{M_{0}}{i k} \frac{\partial}{\partial z}\right)\left[\frac{p}{\zeta}\right]
$$

where $\zeta=\infty$ along the rigid wall part of the GIT, and the unit normal vector to the duct wall is taken as positive when pointing into the fluid. The original form of the above boundary condition contains wall curvature terms. However, these curvature terms have been dropped because the walls in the GIT are considered straight. At the source plane, the acoustic pressure and the transverse component of acoustic particle velocity are assumed known

$$
p=p_{s}(x), \quad v=v_{s}(x)
$$

In general, when sound propagating through the duct reaches the termination located at $z=L$, a radiated and a reflected wave field are produced. This effect is best modeled for an assumed plane wave source by the exit impedance boundary condition

$$
\frac{p}{u}=\rho_{0} c_{0} \zeta_{\text {exit }}
$$


Finally, the Myers boundary condition ${ }^{12}$ assumes the wall impedance is continuous along the liner. This assumption is violated in the GIT due to the abrupt impedance change at the liner leading and trailing edge (see Fig. 1). The LEE model therefore imposes continuity of the acoustic pressure and acoustic particle velocities at the leading and trailing edge of the liner to ensure that the acoustic pressure and velocity field remains a continuum.

\section{B. Convected Helmholtz Equation (CHE) Model}

In the CHE model the mean velocity field is taken as constant $\left(M_{0}(x)=\right.$ constant $)$ so that the acoustic pressure field in the GIT is governed by the convected Helmholtz equation

$$
\left(1-M_{0}^{2}\right) \frac{\partial^{2} p}{\partial z^{2}}+\frac{\partial^{2} p}{\partial x^{2}}-2 i k M_{0} \frac{\partial p}{\partial z}+k^{2} p=0
$$

and the wall impedance boundary condition becomes

$$
-\frac{\partial p}{\partial x}=i k\left[\frac{p}{\zeta}\right]+2 M_{0} \frac{\partial}{\partial z}\left[\frac{p}{\zeta}\right]+\frac{M_{0}^{2}}{i k} \frac{\partial^{2}}{\partial z^{2}}\left[\frac{p}{\zeta}\right]
$$

The sound source boundary condition is

$$
p=p_{s}
$$

and the exit impedance boundary condition becomes

$$
\frac{\partial p}{\partial z}=\frac{-i k p}{M_{0}+\xi_{\text {exit }}}
$$

Finally, the continuity of the acoustic pressure and its gradient at the leading and trailing edge of the liner is imposed to ensure that the acoustic pressure field remains a continuum. Equations (7)-(10) are simply a restatement of Eqs. (3)-(6) under the condition of uniform mean flow.

\section{Solutions to the LEE and CHE Models}

NALYTICAL solutions for the sound field that satisfy the LEE and CHE models can be obtained only for certain A simplified situations. Thus, one must resort to a numerical method that provides approximate, but acceptable solutions. The numerical method chosen to obtain the solution to both models is the conventional finite element method. Both models employ a rectangular element and a uniform mesh. Specific details of the numerical method used in both models are described elsewhere ${ }^{3,5}$ However, to make this text as self-contained as possible, a brief summary of the numerical method for each model is presented.

The convected Helmholtz equation model uses cubic Hermite polynomial basis functions and a weak formulation to satisfy the impedance boundary conditions. The LEE model uses linear polynomials for the basis functions and a strong formulation is used to satisfy the impedance boundary conditions. The sound source boundary condition for both models is implemented by constraining the nodal degrees of freedom at the source plane.

The application of the finite element method to both the CHE and LEE model results in a system of simultaneous linear equations of the form

$$
[A]\{\Phi\}=\{B\}
$$

Options were made available to solve equation (11) using a direct sparse solver for the CHE model and the LAPACK band solver for the LEE model (the sparse solver has not yet been implemented into the LEE model). Both solvers (the direct sparse and LAPACK band solver) run in parallel. The solution for the vector, $\{\Phi\}$, is obtained on the computer platform Lomax. Lomax is an SGI ORIGIN 3000 computer platform with 250 GB RAM and 512 CPUs. Each CPU runs at a speed of $600 \mathrm{MHz}$ and uses the R14000 processor chip.

\section{Impedance Eduction Technique}

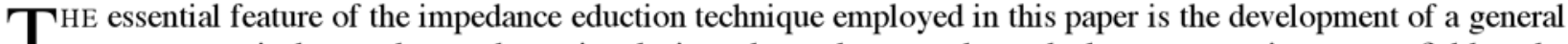
purpose numerical procedure to determine the impedance that reproduces the known acoustic pressure field on the wall opposite the test liner. If the CHE and LEE model are indeed well-posed, then constructing a proof to show that 
the wall impedance of the test specimen is indeed an extrema of the wall error pressure function is relatively straight forward. The procedure used here is to employ the wall error function

$$
F(\zeta)=\sum_{i=1}^{n w a l l}\left[\left.p\left(z_{I}, 0\right)\right|_{\mathrm{FEM}}-\left.p\left(z_{I}, 0\right)\right|_{\text {Meas }}\right]\left[\left.\left.p^{*}\left(z_{I}, 0\right)\right|_{\mathrm{FEM}} p^{*}\left(z_{I}, 0\right)\right|_{\text {Meas }}\right]
$$

as an objective function and determine the unknown impedance such that this objective function is a minimum. The above objective function may be interpreted as the difference between the measured and finite element computed wall pressure opposite the test liner. Note that the proposed procedure is perfectly general and requires only that the CHE and LEE formulation constitute a well-posed boundary value problem. Thus, nonuniform impedance and flows containing shear layers are possible within the context of the current impedance eduction theory. The reader is reminded, however, that the increased generality afforded by the current theory is partly mitigated by the need for a fast and reliable equation solver (equation (11)) and a robust method (i.e., optimizer) for minimizing the objective function.

\section{Optimization Techniques}

$\mathrm{I}^{\mathrm{N}}$ this section three numerical strategies are presented for determining the wall impedance that minimizes the ob1 jective function. The impedance of the test liner is assumed constant so that it consists of a two-parameter family, $\zeta=\theta+i \chi$. Thus, two parameters are free to vary in the optimization: the dimensionless acoustic resistance $(\theta)$ and dimensionless acoustic reactance $(\chi)$.

\section{A. Contour Deformation Method (CDM)}

The most reliable method for determining the minimum in the two-dimensional space defined by the uniform liner is the contour deformation method. In this method the dimensionless resistance is plotted along the abscissa and the dimensionless reactance is plotted along the ordinate. Wall pressure error contours then represent the locus of points in the impedance plane yielding constant values of wall pressure error, $F$. These contours are then plotted using a graphing package. The impedance of the test liner is easily shown to be the location of the center of the contour with the smallest value of wall error. The CDM method is applicable only to a uniform impedance liner. It also has the disadvantage that it is not only computationally expensive, but also labor intensive because a computer-graphics program must be used to map the contours for each frequency and mean flow Mach number. Therefore, two alternative numerical approaches for obtaining the minimum of the wall pressure error function are presented in this paper. These two alternative approaches are more automated and less labor intensive than the contour deformation method. They are also applicable to variable impedance liners.

\section{B. Stewart's Adaptation of Davidon-Fletcher-Powell (SDFP) Method}

The first alternative approach to CDM is the Stewart's adaptation of Davidon-Fletcher-Powell optimization method. ${ }^{13}$ The SDFP is representative of a general class of numerical algorithms known as descent methods. Its defining feature is that the impedance at the minimum point is obtained by iteration with the gradient of the objective being computed numerically by a finite difference approximation. It consists of a process for which the impedance is modified at each cycle of the iteration so that the objective function decreases in value. The iterative process is repeated until convergence to the minimum is achieved to within a specified tolerance. The SDFP combines the best features of steepest decent (good performance if an initial value of the impedance is not close to the minimum point) and Newton's method (rapid convergence if the initial value of impedance is close to the minimum point). The SDFP is generally considered to be a fast, reliable, optimization method and is expected to work well on the objective function used in this report.

Although the convergence is usually rapid, the SDFP algorithm as applied in this paper has the disadvantages of requiring both an initial starting location for the impedance and the computation of the gradient of $F$. In general, the SDFP will locate only local minima. Thus, if the problem has multiple local minima, different initial values of the impedance may iteratively converge to different local minima. Also, when the minimum is located on a relatively flat surface in the function space, the educed impedance may vary because of differences in the convergence tolerance and the direction from which the minimum is approach. Finally, the SDFP is a sequential algorithm that scales poorly on massively parallel computer architectures such as Lomax. 


\section{Genetic Algorithm (GA)}

The second alternative optimization algorithm to the CDM is the Genetic algorithm. ${ }^{14}$ The GA is inspired by Darwin's theory of evolution. Solution for the minimum point using the GA uses an evolutionary process. The algorithm begins with a randomly chosen set of liners called the initial population. The liners from the initial population are taken and used to form a new population of liners. Selection of the new population is motivated by the expectation that the new population will be better (i.e., have a smaller value of wall error) than the old population. Liners that are selected to form the new population (called the offsprings) are therefore selected according to their fitness (i.e., the value of $F$ they produce)- the more suitable they are the more chances they have to reproduce. This reproductive process is repeated for a number of generations or until the condition, $F$ is a minimum, is satisfied within some tolerance.

In contrast to the SDFP, the GA does not require calculation of the gradient of the objective function, nor does it require an initial starting value for the impedance. Furthermore, the GA is a search method that searches the design space for a global minimal point. Thus, the principal drawback of the GA is that many more function evaluations are required when compared to that of SDFP. However, this is more than mitigated by the fact that the Genetic algorithm is highly scalable on massively parallel computing architectures and is normally run in parallel. A more complete description of both the SDFP and GA is beyond the scope of this paper. For a more comprehensive description of both automated search algorithms used in this paper, readers may consult Refs. 13 and 14.

\section{Test Liners and Mean Flow Conditions}

$\mathbf{I}^{\mathrm{N}}$ this paper impedances are educed for a CT65 and a CT57 ceramic tubular test liner. These two test liners are not Itypical of the cavity-backed, perforated face sheet construction presently used for noise abatement. They are chosen because their resistance is dominated by internal fluid viscosity and is thus very insensitive to sound pressure level and mean flow effects, and their impedance spectra vary over a range typical of liners used in aircraft engine nacelles. Figure 2 is a schematic of the CT65 and CT57 ceramic liners. The CT65 liner consists of "sinusoid-shaped" parallel channels embedded in a ceramic matrix. These $77.5 \mathrm{~mm}$-deep channels, with equivalent circular diameters of 0.76 $\mathrm{mm}$, run perpendicular to the exposed surface and provide a surface porosity of 65 percent. The CT57 liner consists of a ceramic structure of parallel, cylindrical channels, embedded in a ceramic matrix. The channels are $85 \mathrm{~mm}$ deep $(d=85.0 \mathrm{~mm})$ and run perpendicular to the exposed surface. The diameter of the cylindrical channels, $a$, is chosen to provide a surface porosity of $\mathbf{5 7}$ percent. For both liners, each channel is rigidly terminated such that it is isolated from its neighbor to ensure a locally reacting structure.

The impedance of each ceramic liner is educed at frequencies for which only the plane wave mode is cut on in the hard wall sections of the GIT. Results are computed for source frequencies ranging from 0.5 to $3.0 \mathrm{kHz}$ in increments of $0.5 \mathrm{kHz}$ and centerline Mach numbers, $M_{c}$, of $0.000,0.100,0.200,0.300,0.475$. A major complication in using GIT data is that in reality the flow contains a shear layer. Therefore, detailed mean flow profiles were acquired at three axial locations ( $z=203 \mathrm{~mm}, z=356 \mathrm{~mm}$, and $z=559 \mathrm{~mm}$ ) and numerical integration was used to compute an average Mach number at each of the three selected axial planes. Finally, the average Mach number for each of the three axial planes were averaged together to obtained a "smeared" average Mach number, $M_{\text {ave }}$, that is used as the uniform flow Mach number for CHE and LEE model. The average Mach number, dimensionless mean pressure, dimensionless mean temperature, and dimensionless mean density measured in the GIT are given in table 1 for each centerline Mach number, $M_{c}$. The mean condition given in table 1 have been nondimensionalized with the constant mean conditions used in Ref. 15. Note that the measured values of mean pressure, mean temperature, and mean density varies somewhat with the centerline Mach number. This contrasts with the assumption used in Ref. 15 for which it was assumed that $T_{0}=T_{\text {ref }}=295.0 \mathrm{~K}, P_{0}=P_{\text {ref }}=101325.0 \mathrm{~Pa}$, and $\rho_{0}=\rho_{\text {ref }}=1.2 \mathrm{~kg} / \mathrm{m}^{3}$. In the result section, a comparison is given of the impedance educed using both the variable mean conditions (table 1) and the constant mean conditions of Ref. 15.

\section{Optimization Parameters}

$\mathrm{F}^{\mathrm{OR}}$ each set of mean flow conditions the impedance is educed using both models (i.e., CHE and LEE model) and F all three optimization techniques (contour deformation (CDM), Stewart's adaptation of Davidon-Fletcher-Powell (SDFP), and the Genetic algorithm (GA)). Although a full description of all parameters used in each optimization method is beyond the scope of this paper a summary of salient features used in each method are summarized below: 


\section{CDM Method}

1. A $201 \times 101$ evenly spaced impedance grid with an increment spacing of $0.05(\Delta \theta=0.05)$ on the dimensionless resistance axis and $0.1(\Delta \chi=0.1)$ on the dimensionless reactance axis

2. Application of Tecplot (version 10) as the graphing package to determine the center of the contour containing the smallest value of $F$

\section{SDFP Method}

1. No constraints on the dimensionless resistance and reactance

2. A central difference approximation to the gradient of $F$

3. An initial impedance of $\zeta=2.0-1.0 i$

4. A tolerance of $10^{-8}$ as the stopping criteria

\section{GA Method}

1. Parallel function evaluations

2. A population density of 100 liners for each generation

3. An evolutionary process using tournament style selection and uniform crossover

4. Creep and jump mutations

5. An elitist strategy

6. Constraints on the dimensionless resistance and reactance $(0.0 \leq \theta \leq 10.0,-10.0 \leq \chi \leq 10.0)$

7. Termination of the evolutionary process after 100 generations

\section{Results and Discussion}

$I^{\mathrm{N}}$

$\mathrm{N}^{\mathrm{N}}$ this section solutions obtained for the unknown impedance from the LEE model are compared to those of the more extensively tested CHE model for the range of frequencies and centerline flow Mach numbers for which measurements in the GIT could be obtained. Impedances are educed using each of the three optimization methods for an assumed plane wave source using the measured termination impedance. One issue which one must come to grips with in this section is how to present the large number of results (two liners, three optimization techniques, four meshes, six frequencies, and six mean flow Mach numbers). Thus, 864 optimization runs were acquired and only a selected number of these results can be presented. To better accommodate the massive number of optimization runs, results are presented in tabular instead of graphical formats. Graphical formats are presented only in a few selected cases for which a graph is appropriate.

\section{A. Parallel Solver Statistics}

Impedances educed for both the CHE and LEE models are obtained on a $65 \times 11$ baseline finite element mesh $(\Delta z=13 \mathrm{~mm}$, and $\Delta x=5 \mathrm{~mm})$. A mesh refinement study is also conducted on three additional meshes to ensure that the educed impedance has converged. The second mesh was obtained by doubling the density of the baseline mesh, the third by doubling the density of the second mesh, and fourth by doubling the density of the third mesh. Thus, the finest mesh has a density eight times finer than the baseline mesh. This subsection presents results from the part of the study concerning solver wall clock time (because Lomax contains $250 \mathrm{~GB}$ of RAM, memory requirements was not an issue).

Table 2 shows the wall clock time in seconds, parallel speedup, and efficiency required to solve the finite element matrix equation (see Eq. (12)) for the sparse and LAPACK band solvers on the smallest mesh. Recall that the CHE model employs the parallel sparse solver, whereas, the LEE model employs the parallel LAPACK band solver. Although the matrix for the sparse solver is 25 percent larger $(N=166,212)$ than that of the LAPACK solver $(N=124,659)$, the sparse solver obtains the solution using considerably less wall clock time. Note also that the parallel LAPACK 
solver uses almost fifty percent more wall clock time on 64 CPUs than on 4 . Similar statistics for the parallel speedup and parallel efficiency are also given in the table. The reference timing for computing the parallel speedup is that required to solve the matrix equation on 4 CPUs (CPUs on Lomax are only allocated in multiples of 4). Note that parallel speedup is obtained for the LAPACK band solver on up to 16 CPUs, whereas, for the sparse solver the parallel speedup is obtained on at least 64 CPUs. Generally speaking, a parallel code is said to be efficient if it runs at 60 percent efficiency or more. Applying this rule of thumb to statistics given in table 2, the parallel solvers run efficiently on up to 8 CPUs. However, since both solvers show parallel speedup (i.e., reduction in wall clock) on as many as $16 \mathrm{CPUs}$, it is reasonable to run both solvers on as many as 16 CPUs to obtain speedup although they would not be running most efficiently. This strategy (running each solver on 16 CPUS) was employed in results obtained in this paper.

In the foregoing paragraph, the statistics related to the parallel equation solvers were discussed. However, for a chosen mesh, the efficiency of the impedance eduction technique depends not only on the choice of solver but also upon the choice of a model. Results in this work show that the CHE model is considerably more efficient in educing the impedance on all four meshes than LEE. This result may reflect the fact that the CHE model has a higher order element (cubic basis functions) than the LEE model (linear basis functions). For example, on the smallest mesh, the average wall clock time to educe the impedance (for each frequency and Mach number) using SDFP and the CHE model was $10.4 \mathrm{~s}$. On the other hand, the average wall clock time required by SDFP using the LEE model was $17.4 \mathrm{~s}$. Reduction in wall clock time on the smallest mesh was much more dramatic for the CHE when compared to that of the LEE model. The average wall clock time to educe the impedance (for a single frequency and Mach number) on the smallest mesh using SDFP with the CHE model was only 1,004 s, whereas, the LEE model required 10,228 s of wall clock time on this mesh. It was also observed that on the smallest mesh the CHE model consumed 1.17 GB of RAM compared to $1.73 \mathrm{~GB}$ for the LEE model. Such large reduction in wall clock time of the CHE model when compared to that of the LEE model cannot be explained by the differences in the efficiency of solvers alone, because each solver was run on 16 CPUs.

\section{B. Performance of Automated Optimizers}

Two different automated optimization algorithms (i.e., GA and SDFP) are used to educe the impedance of the test liner. The performance of the GA and SDFP on the baseline grid for the CHE model is now presented. Although results presented here are for the CT57 liner at Mach 0.255 , they are representative of results obtained over the full range of Mach numbers and liners. Figure 3 shows the performance of SDFP for each frequency. Note that SDFP converges in seven to nine iterations with better performance at the higher frequencies. The wall error function at the anti-resonant frequency $(2.0 \mathrm{kHz})$ is extremely flat and SDFP is known to be deficient on flat surfaces. ${ }^{13}$ Because SDFP use central differences to approximate the gradient of the objective function, approximately nine wall error function evaluations are required per iterations. Thus, the time equivalent of 56 to 81 equation solves are required before SDFP converges.

The Genetic algorithm performance study is presented in Fig. 4. Recall that each population in the GA consists of 100 offsprings. Therefore, the $y$ axis value in Fig. 4 is the wall error corresponding to the offspring with the smallest wall error in the population. Note that the GA converges in ten generations or less and that progress in the minimization of the objective is extremely rapid over the first few generations. Further, the wall clock time required to compute a GA generation is approximately equal to that required to perform an equation solve. It should be also be noted that just as with SDFP, the performance of the GA is better at the higher frequencies (for example, the GA has converged in two generations at $3.0 \mathrm{kHz}$ ).

Generally speaking, in optimum design studies on parallel computing architectures, the most important metric is the wall clock time required to obtain the optimum point. Thus, optimization algorithms that minimize the wall clock time are normally preferred. Figures 3 and 4 provide insights as to which of the automated optimization algorithms returns the educed impedance within the smallest wall clock time on Lomax. Note that the GA returns the optimum point in a wall clock time that is nearly an order of magnitude less than SDFP. On the other hand, the GA could not be run in parallel on a sequential computing platform and would require $20 \%$ more wall clock time than the GA on a sequential computer platform.

\section{Infinitely Long CT65 Liner Results}

In the first example, the LEE model is validated in a 51-mmx51-mm duct for an infinitely long CT65 liner, for which the exact zero flow impedance was known from normal incidence impedance tube measurements. For simplicity, attention is focused only on downstream propagation of the lowest order mode in the infinite duct. Source plane data, exit impedance, and lower wall acoustic pressure data required to educe the impedance is synthesized from the exact 
mode solution in an infinitely long duct. Figure 5 compares the dimensionless impedance educed using the CHE model with the exact values using SDFP. Results are plotted on a dual axis system with resistance plotted along the $y 1$ axis and reactance along the $y 2$ axis. Because the CHE model educed the same impedance for each Mach number on each of the four meshes, only a single curve is shown. The numerically educed impedance from SDFP is in full agreement with the exact value. SDFP results using the LEE model were also computed on each mesh, and results are given in table 3. Good agreement between the exact and educed impedances for each mesh is observed, with one exception. The baseline mesh (i.e., the coarsest mesh) has not converged to the correct impedance. This simply reflects the fact that the baseline mesh is too coarse for the impedance eduction process to be accurate.

Before proceeding to the next example, it is worth noting that the Genetic algorithm and the contour deformation method were applied to the infinitely long CT65 data. The educed impedances are not presented for the sake of brevity. However, the educed resistance and reactance from these two optimization algorithms were in agreement with that obtained by SDFP to within \pm 0.05 .

\section{Finite Length CT57 Liner}

In this second example the impedance of the CT57 liner is educed using test data acquired at $140 \mathrm{~dB}$ reference sound pressure level in the GIT with the test liner installed. This example allows one to evaluate the models in the presence of real test data, with abrupt transitions at the leading and trailing edge of the liner. Educed resistances for the CT57 liner on the second mesh are given in table 4 , and the corresponding reactances are given in table 5 . The coarsest mesh results did not converge for the LEE model, but the second, third, and fourth mesh converged to nearly identical impedances. Therefore, only second mesh results are presented in the table (the CHE model converged for all meshes). Educed impedances are given for both the convected Helmholtz and the linearized Euler models and for each of the three optimization techniques. Results computed in Ref. 15 are also presented in the table. Recall that the Ref. 15 results assumed the same set of mean conditions $\left(T_{0}=T_{r e f}, P_{0}=P_{r e f}\right.$, and $\left.\rho_{0}=\rho_{r e f}\right)$ for each mean flow Mach number. However, table 1 shows that these mean conditions change with the centerline Mach number.

Several observations are noted from the CT57 educed impedances tabulated in tables 4 and 5. Note that for each optimization method, the convected Helmholtz and Linearized Euler model educe essentially the same resistance and reactance at each frequency and flow Mach number with one exception. The two models educed different impedances at $0.5 \mathrm{kHz}$ for high Mach numbers. This anomaly has been reported in previous papers and for different liners. It shows up here for the CT57 liner in the frequency and Mach number range (i.e., low frequency and high Mach number) for which shear layer effects are generally thought to be most important. Note also that the $0.5 \mathrm{kHz}$ anomaly is not present at low Mach number and becomes more and more dominant as the flow speed increases. Another important observation is that the results of Ref. 15 agree with results in this paper except where the anomaly occurs (i.e., 0.5 $\mathrm{kHz}$ and high Mach number) and the anti-resonant peak (i.e., $2.0 \mathrm{kHz}$ ).

Figures $6,7,8$, and 9 show contour maps of the reciprocal of the wall error function $(1 / F)$ at $0.5 \mathrm{kHz}$ for increasing flow speeds. The contour maps were produced from the convected Helmholtz model. Note that at zero Mach number (Fig. 6) the contour map has a unique, clearly defined eye that contains the maximum of $1 / F$ (i.e., the minimum of $F$ ). As Mach number increases (Fig. 7, 8, and 9) the eye becomes more and more diffused and the surface in the vicinity of the minimum is extremely flat with a diameter of approximately $1.2 \rho_{0} c_{0}$ at the highest Mach number. Thus, errors in educed dimensionless resistance and reactance of 1.2 would not be unusual at this frequency. In all likelihood, the $0.5 \mathrm{kHz}$ contours at the high Mach numbers are non-physical and probably generated by the absence of the shear layer that should be included in future computations.

The effects of the duct termination on the educed resistance and reactance of the CT57 liner are given in table 6a and $6 \mathrm{~b}$, respectively. Here the CHE model was used to evaluate the effects of the measured termination and for an assumed anechoic termination using SDFP. Results in the table were computed on the second mesh. It is clear from these computations that both terminations give nearly identical educed impedances except at high Mach number near the sharp anti-resonant peak. Results similar to those of table 6 were found for the LEE model.

\section{Concluding Remarks}

This paper has presented a comparison of the impedances educed from a linearized Euler model to that of a welltested convected Helmholtz model, under the assumption of a uniform mean flow field and a plane wave source. Test data was acquired from ceramic tubular test samples installed in the NASA Langley Grazing Incidence Tube. Impedances were educed using three search techniques (the contour deformation method, Stewart's adaptation of the Davidon-Fletcher-Powell method, and the Genetic Algorithm) for the range of frequencies and centerline Mean flow 
Mach numbers for which data consistent with the plane wave assumption could be acquired. The principal findings of this paper are

1. All three optimization techniques consistently converged to the same impedance for all frequencies and average flow Mach numbers when used with the same model.

2. Nearly identical impedances are obtained from the two models, with the exception that different impedances were educed at $0.5 \mathrm{kHz}$ at high mean flow speeds.

3. The use of an anechoic termination with measured data in the NASA Langley Grazing Incidence Tube is valid except near a sharp anti-resonant peak.

Consistency between the impedances educed using the two models also provides confidence that the linearized Euler model is ready for application to more realistic flows, such as those containing shear layers. Finally, because the 0.5 $\mathrm{kHz}$ anomaly occurs at low frequency where the shear layers is known to be important, it is likely due to absence of the shear layer.

\section{References}

${ }^{1}$ Armstrong, D. L., “Acoustic Grazing Flow Impedance Using Waveguide Principles," NASA CR-120848, 1971.

${ }^{2}$ Watson, W. R., Jones, M. G., Tanner, S. E., and Parrott, T. L., “ A Finite Element Propagation Model for Extracting Normal Incidence Impedance in Nonprogressive Acoustic Wave Fields," Journal of Computational Physics, Vol. 125, No. 1, April 1996, pp.177 - 186.

${ }^{3}$ Watson, W. R., Jones, M. G., and Parrott, T. L., "Validation of an Impedance Eduction Method in Flow," AIAA Journal Vol. 37, No. 7, July 1999, pp. 818-824.

${ }^{4}$ Watson, W., Jones, M., and Parrott, T., " A Quasi-3-D Theory for Impedance Eduction in Uniform Grazing Flow," AIAA Paper 2005-2848, Proceedings of the 11th AIAA/CEAS Aeroacoustics Conference \& Exhibit, May 23 - 25, 2005, Monterey, CA.

${ }^{5}$ Watson, W. R., Tracy, M. B., Jones, M. G., and Parrott, T. L., "Impedance Eduction in Shear Flow," AIAA Paper 2001-2263, Proceedings of the 7th AIAA/CEAS Aeroacoustics Conference \& Exhibit, Maastricht, The Netherlands, May 28 - 30, 2001.

${ }^{6}$ Jones, M. G., Parrott, T. L., and Watson, W. R., "Comparison of Acoustic Impedance Eduction Techniques for Locally-Reacting Liners," AIAA Paper 2002-2246, Proceedings of the 8th AIAA/CEAS Aeroacoustics Conference \& Exhibit, Breckenridge, CO, June 17 - 19, 2002.

${ }^{7}$ Jones, M. G., Watson, W. R., and Parrott, T. L., “Design and Evaluation of Modifications to the NASA Langley Flow Impedance Tube," AIAA Paper 2004-2837, Proceedings of the 10th AIAA/CEAS Aeroacoustics Conference \& Exhibit, Manchester, United Kingdom, May $12-14$, 2004.

${ }^{8}$ Ozyoruk, Y., Long, L. N., and Jones, M. G.,“Time-Domain Numerical Simulations of a Flow-Impedance Tube,”Journal Of Computational Physics, Vol. 146, pp. 29-57, 1998.

${ }^{9}$ Ozyoruk, Y., and Long, L. N.,"Time-Domain Calculations of Sound Propagation in Lined Ducts with Sheared Flows," AIAA Journal, Vol. 38 , pp. 768-773, 2001.

${ }^{10} \mathrm{Ju}, \mathrm{H}$., and Fung, K. Y., “Time-Domain Impedance Boundary Conditions with Mean Flow Effects," AIAA Journal, Vol. 39, pp. 1683-1690, 2001.

${ }^{11}$ Zheng, S., and Zhuang, M.,"Verification and Validation of Time-Domain Impedance Boundary Condition in Lined Ducts," AIAA Journal, Vol. 43, pp. 306-313, 2005

${ }^{12}$ Myers, M.K.,"On The Acoustic Boundary Condition In The Presence Of Flow," Journal Of Sound And Vibrations, Vol. 71, No. 3, 1980, pp. $429-434,1980$

${ }^{13}$ Stewart, G.W. III, “A Modification of Davidon's Minimization Method to Accept Difference Approximations of Derivatives," Journal of ACM, Vol. 14, No. 1, Jan. 1967, pp. 72-83.

${ }^{14}$ Goldberg, D., Genetic Algorithms in Search, Optimization and Machine Learning, Addison-Wesley, New York, NY, 1989 , pp. $75-147$.

${ }^{15}$ Jones, M., Watson, W., and Parrott, T., “ Benchmark Data for Evaluation of Aeroacoustic Propagation Codes with Grazing Flow," AIAA Paper 2005-2853, Proceedings of the 12th AIAA/CEAS Aeroacoustics Conference, May 23-25, 2005, Monterey, CA. 


\section{Tables and Figures}

Table 1. Measured Mean Flow Condition in the GIT

$$
\left(T_{r e f}=295.0 \mathrm{~K}, P_{r e f}=101325.0 \mathrm{~Pa} \text {, and } \rho_{r e f}=1.2 \mathrm{~kg} / \mathrm{m}^{3}\right)
$$

\begin{tabular}{|c|c|c|c|c|}
\hline$M_{c}$ & $M_{\text {ave }}$ & $P_{0} / P_{\text {ref }}$ & $T_{0} / T_{\text {ref }}$ & $\rho_{0} / \rho_{\text {ref }}$ \\
\hline 0.000 & 0.000 & 1.003 & 1.002 & 1.001 \\
0.100 & 0.079 & 0.982 & 1.006 & 0.976 \\
0.200 & 0.172 & 1.021 & 1.004 & 1.017 \\
0.30 & 0.255 & 1.061 & 1.005 & 1.056 \\
0.400 & 0.335 & 1.130 & 1.001 & 1.129 \\
0.475 & 0.400 & 1.129 & 1.004 & 1.125 \\
\hline
\end{tabular}

Table 2. Sparse and LAPACK Solver Statistics for the Solution of $[A]\{\Phi\}=\{B\}$ on the Finest Mesh

\begin{tabular}{|c|c|c|c|c|c|c|}
\hline $\begin{array}{c}\text { Number } \\
\text { of CPUs }\end{array}$ & $\begin{array}{c}\text { Sparse } \\
\text { Wall Clock } \\
N=166,212\end{array}$ & $\begin{array}{c}\text { LAPACK } \\
\text { Wall Clock } \\
N=124,659\end{array}$ & $\begin{array}{c}\text { Sparse } \\
\text { Speedup } \\
N=166,212\end{array}$ & $\begin{array}{c}\text { LAPACK } \\
\text { Speedup } \\
N=124,659\end{array}$ & $\begin{array}{c}\text { Sparse } \\
\text { Efficiency } \\
N=166,212\end{array}$ & $\begin{array}{c}\text { LAPACK } \\
\text { Efficiency } \\
N=124,659\end{array}$ \\
\hline 4 & $36 \mathrm{~s}$ & $74 \mathrm{~s}$ & 1.00 & 1.00 & $100 \%$ & $100 \%$ \\
8 & $28 \mathrm{~s}$ & $60 \mathrm{~s}$ & 1.29 & 1.23 & $65 \%$ & $62 \%$ \\
16 & $26 \mathrm{~s}$ & $65 \mathrm{~s}$ & 1.35 & 1.14 & $34 \%$ & $29 \%$ \\
32 & $27 \mathrm{~s}$ & $77 \mathrm{~s}$ & 1.33 & 0.96 & $17 \%$ & $12 \%$ \\
64 & $32 \mathrm{~s}$ & $108 \mathrm{~s}$ & 1.13 & 0.68 & $7 \%$ & $4 \%$ \\
\hline
\end{tabular}




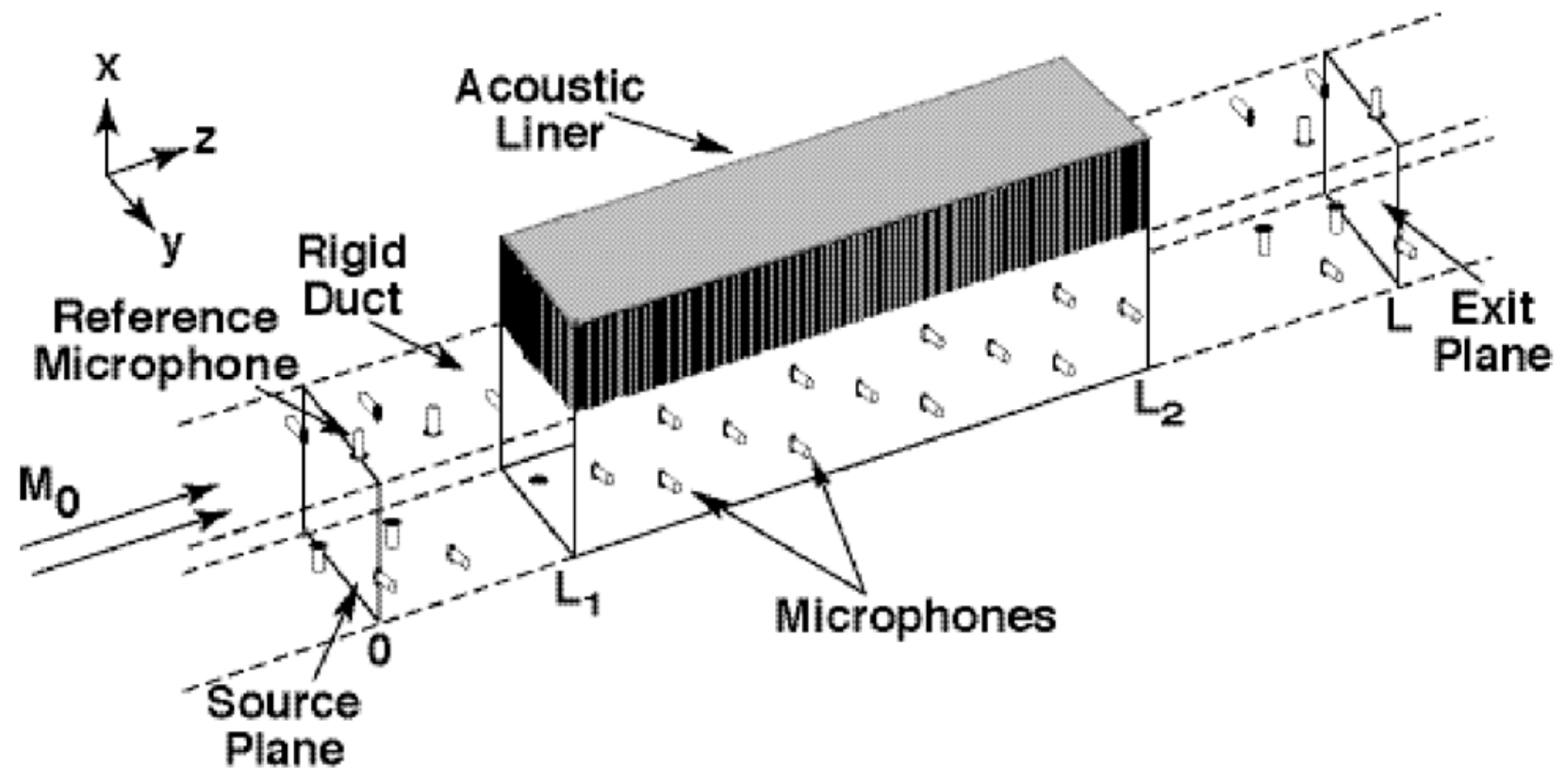

Figure 1. Schematic of LaRC's Grazing Incidence Tube. 


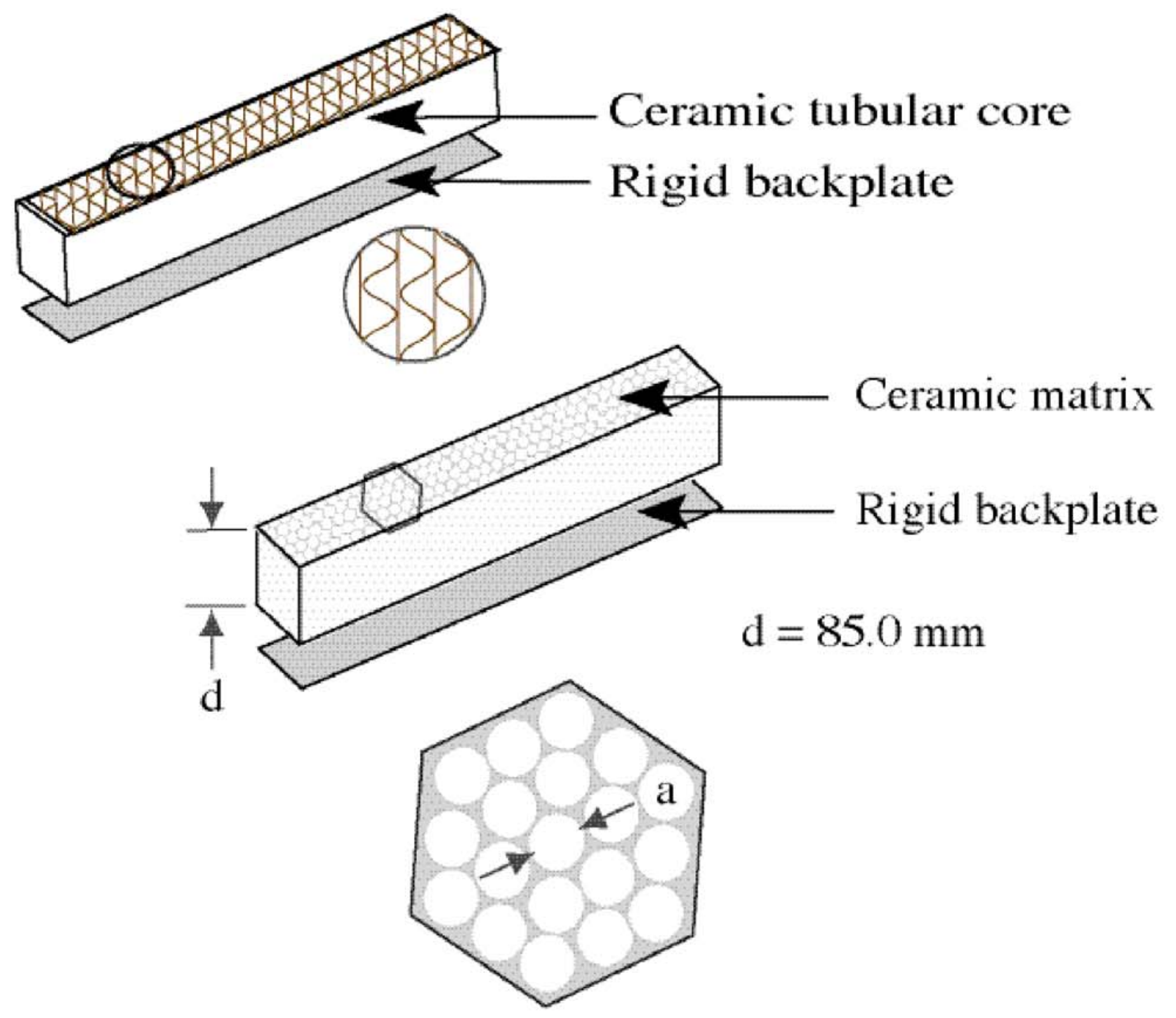

Figure 2. Schematic of ceramic tubular test liners. 
Table 3. SDFP educed impedance from LEE Model for CT65 liner

\begin{tabular}{|c|c|c|c|c|c|c|c|c|c|c|c|}
\hline$M_{\text {ave }}$ & $\mathrm{kHz}$ & \multicolumn{2}{|c|}{ Exact } & \multicolumn{2}{|c|}{ Mesh 1} & \multicolumn{2}{|c|}{ Mesh 2} & \multicolumn{2}{|c|}{ Mesh 3} & \multicolumn{2}{|c|}{ Mesh 4} \\
\hline & & $\theta$ & $\chi$ & $\theta$ & $\chi$ & $\theta$ & $\chi$ & $\theta$ & $\chi$ & $\theta$ & $x$ \\
\hline 0.000 & 0.5 & .44 & 0.66 & .45 & .65 & 0.44 & 0.66 & 0.44 & 0.66 & .44 & -0.66 \\
\hline 0.079 & & 14 & 0.66 & 47 & .65 & 45 & .66 & 45 & 0.66 & & .66 \\
\hline 0.172 & 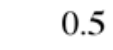 & 14 & -0.66 & .49 & 65 & 46 & -0.66 & .45 & -0.66 & & 0.66 \\
\hline 0.255 & 5 & 44 & -0.66 & .60 & 0.71 & 48 & -0.69 & .44 & -0.62 & 45 & -0.65 \\
\hline 0. & 5 & 14 & -0.66 & 0.30 & .90 & & -0.72 & 0.47 & -0.67 & & -0.66 \\
\hline 0.400 & 5 & 44 & -0.66 & .52 & 0.51 & 48 & -0.63 & .46 & -0.65 & .45 & -0.65 \\
\hline 0.000 & & 40 & -0.61 & 0.42 & .57 & & -0.60 & 0.40 & -0.61 & .40 & -0.61 \\
\hline 0.079 & & & -0.61 & 15 & & & .61 & 41 & -0.61 & & -0.61 \\
\hline 0.172 & 0 & 10 & -0.61 & 0.48 & -0.59 & & -0.61 & .42 & -0.61 & 41 & -0.61 \\
\hline & & & -0.61 & & & & 59 & & -0.60 & & .61 \\
\hline 0 & & 10 & -0.61 & 0 & -0.52 & & -0.57 & .43 & -0.59 & 10 & -0.61 \\
\hline 0.400 & & & -0.61 & & & & -0.60 & 43 & -0.59 & & -0.60 \\
\hline 0.000 & & & 2.48 & & 245 & & 2.48 & 0.63 & 2.48 & & 2.48 \\
\hline 0.079 & & & & & 2.46 & & 48 & 63 & 48 & & .48 \\
\hline 0.172 & & & 2.48 & 0 & 249 & & 2.49 & 61 & 2.49 & & 2.48 \\
\hline & & & & & & & 50 & 50 & 49 & & .49 \\
\hline 0 & & & & & & & 2.50 & 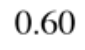 & 49 & & 2.49 \\
\hline 0.400 & & & & & & & 51 & 58 & .49 & & 2.48 \\
\hline 0.00 & & & & & & & -1.46 & & & & -1.47 \\
\hline 0.079 & & 3 & 17 & & 39 & & -1.45 & 75 & -1.46 & 74 & -1.47 \\
\hline 0.172 & & 3 & & & & & -1.45 & 0.77 & -1.46 & & -1.46 \\
\hline 0.255 & & 0.73 & -1.47 & & -1.34 & 33 & -1.44 & 0.78 & -1.45 & 76 & -1.46 \\
\hline & & & & & & & & & & & -1.47 \\
\hline 0.400 & & 0.73 & -1.47 & 1.18 & -1.30 & 1.04 & -1.59 & 0.72 & -1.47 & 0.75 & -1.48 \\
\hline & & & & & & & & & & & \\
\hline 0.079 & & 15 & & 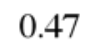 & 5 & 6 & 18 & .45 & 17 & 5 & 0.17 \\
\hline & & & & & & & & & & & \\
\hline 0.255 & & 0 & 7 & 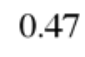 & 8 & 15 & 0.18 & 0.45 & 18 & 45 & 0.17 \\
\hline & & & & & & & -1.60 & & & & 0.17 \\
\hline 0.400 & & 0.45 & 17 & 0.49 & 0.20 & 0.45 & 0.19 & 0.45 & 0.18 & 0.45 & 0.18 \\
\hline & & & & & & & 1.22 & 0.50 & 1.29 & 0. & 1.30 \\
\hline 0.079 & & 0.49 & & & & & 1.27 & 0.49 & 1.31 & & 1.30 \\
\hline & & & & & & & & 0.48 & 31 & & 1.31 \\
\hline & & 0.49 & & & & & 1.32 & 0.46 & 1.31 & & 1.31 \\
\hline & & & & & & & & 0.46 & 1.32 & 0.47 & 1.31 \\
\hline 0.400 & 3.0 & 0.49 & 1.30 & 0.47 & 1.04 & 0.46 & 1.33 & 0.46 & 1.32 & 0.47 & 1.31 \\
\hline
\end{tabular}


Table 4. Educed Resistance for the CT57 Liner on the Second Mesh

\begin{tabular}{|c|r|r|r|r|r|r|r|r|}
\hline$M_{\text {ave }}$ & $f, \mathrm{kHz}$ & \multicolumn{3}{|c|}{ Convected Helmholtz } & \multicolumn{3}{|c|}{ Linearized Euler } \\
\hline & & GA & SDFP & CDM & Ref. 15 & GA & SDFP & CDM \\
\hline 0.000 & 0.5 & 0.55 & 0.53 & 0.53 & 0.58 & 0.55 & 0.53 & 0.53 \\
0.000 & 1.0 & 0.47 & 0.49 & 0.48 & 0.49 & 0.47 & 0.49 & 0.49 \\
0.000 & 1.5 & 1.18 & 1.16 & 1.15 & 1.12 & 1.18 & 1.16 & 1.15 \\
0.000 & 2.0 & 5.12 & 5.12 & 5.10 & 4.75 & 5.04 & 5.08 & 5.09 \\
0.000 & 2.5 & 1.50 & 1.47 & 1.48 & 1.49 & 1.50 & 1.47 & 1.45 \\
0.000 & 3.0 & 0.71 & 0.75 & 0.74 & 0.74 & 0.71 & 0.75 & 0.75 \\
\hline 0.079 & 0.5 & 0.71 & 0.71 & 0.71 & 0.77 & 0.63 & 0.63 & 0.66 \\
0.079 & 1.0 & 0.39 & 0.40 & 0.41 & 0.39 & 0.47 & 0.44 & 0.42 \\
0.079 & 1.5 & 1.10 & 1.08 & 1.09 & 1.00 & 1.10 & 1.09 & 1.07 \\
0.079 & 2.0 & 4.09 & 4.10 & 4.08 & 3.69 & 4.09 & 4.12 & 4.11 \\
0.079 & 2.5 & 1.42 & 1.38 & 1.37 & 1.45 & 1.42 & 1.38 & 1.39 \\
0.079 & 3.0 & 0.71 & 0.70 & 0.71 & 0.68 & 0.71 & 0.72 & 0.71 \\
\hline 0.172 & 0.5 & 0.63 & 0.79 & 0.80 & 0.85 & 0.63 & 0.63 & 0.62 \\
0.172 & 1.0 & 0.32 & 0.33 & 0.34 & 0.33 & 0.39 & 0.41 & 0.43 \\
0.172 & 1.5 & 1.26 & 1.26 & 1.26 & 1.22 & 1.26 & 1.27 & 1.25 \\
0.172 & 2.0 & 4.96 & 4.94 & 4.96 & 4.75 & 4.96 & 4.95 & 4.94 \\
0.172 & 2.5 & 1.10 & 1.11 & 1.10 & 1.15 & 1.10 & 1.12 & 1.11 \\
0.172 & 3.0 & 0.79 & 0.81 & 0.80 & 0.79 & 0.87 & 0.83 & 0.85 \\
\hline 0.255 & 0.5 & 0.63 & 0.62 & 0.63 & 0.64 & 0.47 & 0.45 & 0.47 \\
0.255 & 1.0 & 0.24 & 0.27 & 0.26 & 0.27 & 0.39 & 0.39 & 0.40 \\
0.255 & 1.5 & 1.26 & 1.24 & 1.24 & 1.18 & 1.26 & 1.23 & 1.22 \\
0.255 & 2.0 & 5.35 & 5.33 & 5.32 & 5.31 & 5.35 & 5.35 & 5.33 \\
0.255 & 2.5 & 0.94 & 0.97 & 0.99 & 1.02 & 0.94 & 0.98 & 1.00 \\
0.255 & 3.0 & 0.79 & 0.76 & 0.75 & 0.67 & 0.79 & 0.78 & 0.80 \\
\hline 0.335 & 0.5 & 0.71 & 0.69 & 0.70 & 0.70 & 0.32 & 0.03 & 0.31 \\
0.335 & 1.0 & 0.24 & 0.20 & 0.20 & 0.20 & 0.39 & 0.36 & 0.38 \\
0.335 & 1.5 & 1.18 & 1.19 & 1.20 & 1.19 & 1.18 & 1.17 & 1.18 \\
0.335 & 2.0 & 4.57 & 4.60 & 4.59 & 4.81 & 4.17 & 4.20 & 4.19 \\
0.335 & 2.5 & 0.94 & 0.91 & 0.90 & 0.92 & 0.87 & 0.90 & 0.93 \\
0.335 & 3.0 & 0.71 & 0.73 & 0.72 & 0.72 & 0.79 & 0.77 & 0.78 \\
\hline 0.400 & 0.5 & 0.87 & 0.86 & 0.88 & 0.88 & 0.47 & 0.01 & 0.47 \\
0.400 & 1.0 & 0.08 & 0.07 & 0.05 & 0.07 & 0.32 & 0.30 & 0.29 \\
0.400 & 1.5 & 1.26 & 1.26 & 1.27 & 1.23 & 1.34 & 1.32 & 1.30 \\
0.400 & 2.0 & 2.44 & 2.47 & 2.45 & 2.77 & 2.44 & 2.41 & 2.40 \\
0.400 & 2.5 & 0.79 & 0.80 & 0.79 & 0.84 & 0.79 & 0.78 & 0.79 \\
0.400 & 3.0 & 0.79 & 0.75 & 0.76 & 0.66 & 0.79 & 0.78 & 0.79 \\
\hline
\end{tabular}


Table 5. Educed Reactance for the CT57 Liner on the Second Mesh

\begin{tabular}{|c|c|c|c|c|c|c|c|c|}
\hline \multirow[t]{2}{*}{$M_{\text {ave }}$} & \multirow[t]{2}{*}{$f, \mathrm{kHz}$} & \multicolumn{4}{|c|}{ Convected Helmholtz } & \multicolumn{3}{|c|}{ Linearized Euler } \\
\hline & & GA & SDFP & CDM & Ref. 15 & GA & SDFP & $\mathrm{CDM}$ \\
\hline 0.000 & 0.5 & -1.74 & -1.70 & -1.70 & -1.74 & -1.69 & -1.70 & -1.69 \\
\hline 0.000 & 1.0 & 0.12 & 0.13 & 0.13 & 0.13 & 0.12 & 0.13 & 0.13 \\
\hline 0.000 & 1.5 & 1.29 & 1.28 & 1.27 & 1.29 & 1.27 & 1.28 & 1.27 \\
\hline 0.000 & 2.0 & 0.90 & 0.88 & 0.88 & 0.81 & 0.96 & 0.85 & 0.84 \\
\hline 0.000 & 2.5 & -1.61 & -1.59 & -1.58 & -1.59 & -1.61 & -1.58 & -1.59 \\
\hline 0.000 & 3.0 & -0.20 & -0.18 & -0.19 & -0.19 & -0.20 & -0.19 & -0.18 \\
\hline 0.079 & 0.5 & -1.58 & -1.55 & -1.55 & -1.58 & -1.61 & -1.59 & -1.58 \\
\hline 0.079 & 1.0 & 0.04 & 0.08 & 0.09 & 0.08 & 0.04 & 0.07 & 0.08 \\
\hline 0.079 & 1.5 & 1.29 & 1.27 & 1.28 & 1.26 & 1.22 & 1.24 & 1.22 \\
\hline 0.079 & 2.0 & 0.98 & 1.00 & 0.98 & 1.17 & 0.90 & 0.92 & 0.91 \\
\hline 0.079 & 2.5 & -1.45 & -1.48 & -1.47 & -1.45 & -1.45 & -1.47 & -1.46 \\
\hline 0.079 & 3.0 & -0.27 & -0.26 & -0.26 & -0.28 & -0.27 & -0.26 & -0.27 \\
\hline 0.172 & 0.5 & -1.50 & -1.49 & -1.49 & -1.50 & -1.61 & -1.63 & -1.62 \\
\hline 0.172 & 1.0 & 0.20 & 0.17 & 0.16 & 0.17 & 0.12 & 0.15 & 0.15 \\
\hline 0.172 & 1.5 & 1.29 & 1.31 & 1.30 & 1.31 & 1.22 & 1.25 & 1.24 \\
\hline 0.172 & 2.0 & -0.51 & -0.54 & -0.55 & -0.21 & -0.67 & -0.67 & -0.68 \\
\hline 0.172 & 2.5 & -1.45 & -1.48 & -1.49 & -1.47 & -1.45 & -1.49 & -1.48 \\
\hline 0.172 & 3.0 & -0.12 & -0.14 & -0.15 & -0.15 & -0.20 & -0.16 & -0.17 \\
\hline 0.255 & 0.5 & -1.06 & -1.07 & -1.05 & -1.09 & -1.37 & -1.35 & -1.33 \\
\hline 0.255 & 1.0 & 0.12 & 0.11 & 0.10 & 0.12 & 0.04 & 0.07 & 0.08 \\
\hline 0.255 & 1.5 & 1.37 & 1.38 & 1.39 & 1.37 & 1.29 & 1.32 & 1.30 \\
\hline 0.255 & 2.0 & -1.37 & -1.34 & -1.34 & -1.05 & -1.45 & -1.45 & -1.47 \\
\hline 0.255 & 2.5 & -1.45 & -1.44 & -1.45 & -1.44 & -1.45 & -1.48 & -1.50 \\
\hline 0.255 & 3.0 & -0.20 & -0.20 & -0.20 & -0.12 & -0.20 & -0.21 & -0.20 \\
\hline 0.335 & 0.5 & -0.59 & -0.56 & -0.55 & -0.57 & -0.98 & -1.31 & -1.30 \\
\hline 0.335 & 1.0 & 0.12 & 0.15 & 0.15 & 0.15 & 0.04 & 0.07 & 0.08 \\
\hline 0.335 & 1.5 & 1.29 & 1.32 & 1.32 & 1.31 & 1.29 & 1.30 & 1.29 \\
\hline 0.335 & 2.0 & -1.69 & -1.67 & -1.67 & -1.62 & -1.69 & -1.70 & -1.69 \\
\hline 0.335 & 2.5 & -1.37 & -1.39 & -1.40 & -1.39 & -1.45 & -1.46 & -1.46 \\
\hline 0.335 & 3.0 & -0.20 & -0.23 & -0.24 & -0.23 & -0.27 & -0.24 & 0.24 \\
\hline 0.400 & 0.5 & -0.35 & -0.34 & -0.35 & -0.35 & -0.75 & -1.01 & -1.00 \\
\hline 0.400 & 1.0 & 0.12 & 0.14 & 0.12 & 0.14 & 0.04 & 0.05 & 0.07 \\
\hline 0.400 & 1.5 & 1.14 & 1.16 & 1.13 & 1.19 & 1.22 & 1.19 & 1.20 \\
\hline 0.400 & 2.0 & -2.63 & -2.63 & -2.65 & -2.63 & -2.47 & -2.43 & -2.40 \\
\hline 0.400 & 2.5 & -1.29 & -1.25 & -1.23 & -1.25 & -1.29 & -1.27 & -1.26 \\
\hline 0.400 & 3.0 & -0.27 & -0.24 & -0.23 & -0.23 & -0.27 & -0.28 & -0.27 \\
\hline
\end{tabular}


Table 6a. Educed Resistance for the CT57 liner using the Measured and Anechoic Termination

\begin{tabular}{|c|c|c|c|c|c|c|c|c|c|c|c|c|}
\hline f, kHz & \multicolumn{2}{|c|}{$M_{0}=0.000$} & \multicolumn{2}{c|}{$M_{0}=0.079$} & \multicolumn{2}{c|}{$M_{0}=0.172$} & \multicolumn{2}{c|}{$M_{0}=0.255$} & \multicolumn{2}{c|}{$M_{0}=0.355$} & \multicolumn{2}{c|}{$M_{0}=0.400$} \\
\hline & Meas & $\rho_{0} c_{0}$ & Meas & $\rho_{0} c_{0}$ & Meas & $\rho_{0} c_{0}$ & Meas & $\rho_{0} c_{0}$ & Meas & $\rho_{0} c_{0}$ & Meas & $\rho_{0} c_{0}$ \\
\hline 0.5 & 0.53 & 0.51 & 0.63 & 0.62 & 0.63 & 0.65 & 0.45 & 0.43 & 0.35 & 0.36 & 0.43 & 0.43 \\
1.0 & 0.49 & 0.49 & 0.44 & 0.44 & 0.41 & 0.41 & 0.39 & 0.39 & 0.36 & 0.36 & 0.30 & 0.30 \\
1.5 & 1.16 & 1.15 & 1.09 & 1.08 & 1.27 & 1.27 & 1.23 & 1.23 & 1.17 & 1.22 & 1.32 & 1.35 \\
2.0 & 5.08 & 4.97 & 4.12 & 4.08 & 4.95 & 4.98 & 5.35 & 5.30 & 4.20 & 4.51 & 2.40 & 2.45 \\
2.5 & 1.47 & 1.46 & 1.38 & 1.38 & 1.12 & 1.12 & 0.98 & 0.98 & 0.90 & 0.90 & 0.78 & 0.78 \\
3.0 & 0.75 & 0.75 & 0.72 & 0.72 & 0.83 & 0.83 & 0.78 & 0.78 & 0.77 & 0.77 & 0.78 & 0.78 \\
\hline
\end{tabular}

Table 6b. Educed Reactance for the CT57 Liner using the Measured and Anechoic Termination

\begin{tabular}{|r|r|r|r|r|r|r|r|r|r|r|r|r|}
\hline f,kHz & \multicolumn{2}{|c|}{$M_{0}=0.000$} & \multicolumn{2}{c|}{$M_{0}=0.079$} & \multicolumn{2}{c|}{$M_{0}=0.172$} & \multicolumn{2}{c|}{$M_{0}=0.255$} & \multicolumn{2}{c|}{$M_{0}=0.355$} & \multicolumn{2}{|c|}{$M_{0}=0.400$} \\
\hline & \multicolumn{1}{|r|}{ Meas } & $\rho_{0} c_{0}$ & Meas & $\rho_{0} c_{0}$ & Meas & $\rho_{0} c_{0}$ & Meas & $\rho_{0} c_{0}$ & Meas & $\rho_{0} c_{0}$ & Meas & $\rho_{0} c_{0}$ \\
\hline 0.5 & -1.70 & -1.69 & -1.59 & -1.59 & -1.63 & -1.63 & -1.35 & -1.45 & -0.96 & -0.95 & -0.76 & -0.79 \\
1.0 & 0.13 & 0.13 & 0.07 & 0.07 & 0.15 & 0.15 & 0.07 & 0.07 & 0.07 & 0.07 & 0.05 & 0.05 \\
1.5 & 1.28 & 1.29 & 1.24 & 1.26 & 1.25 & 1.25 & 1.32 & 1.32 & 1.30 & 1.29 & 1.19 & 1.22 \\
2.0 & 0.85 & 0.86 & 0.92 & 0.97 & -0.67 & -0.75 & -1.45 & -1.79 & -1.70 & -1.90 & -2.43 & -2.49 \\
2.5 & -1.58 & -1.58 & -1.47 & -1.47 & -1.49 & -1.49 & -1.48 & -1.48 & -1.46 & -1.46 & -1.27 & -1.27 \\
3.0 & -0.19 & -0.19 & -0.26 & -0.26 & -0.16 & -0.16 & -0.21 & -0.21 & -0.24 & -0.24 & -0.28 & -0.28 \\
\hline
\end{tabular}




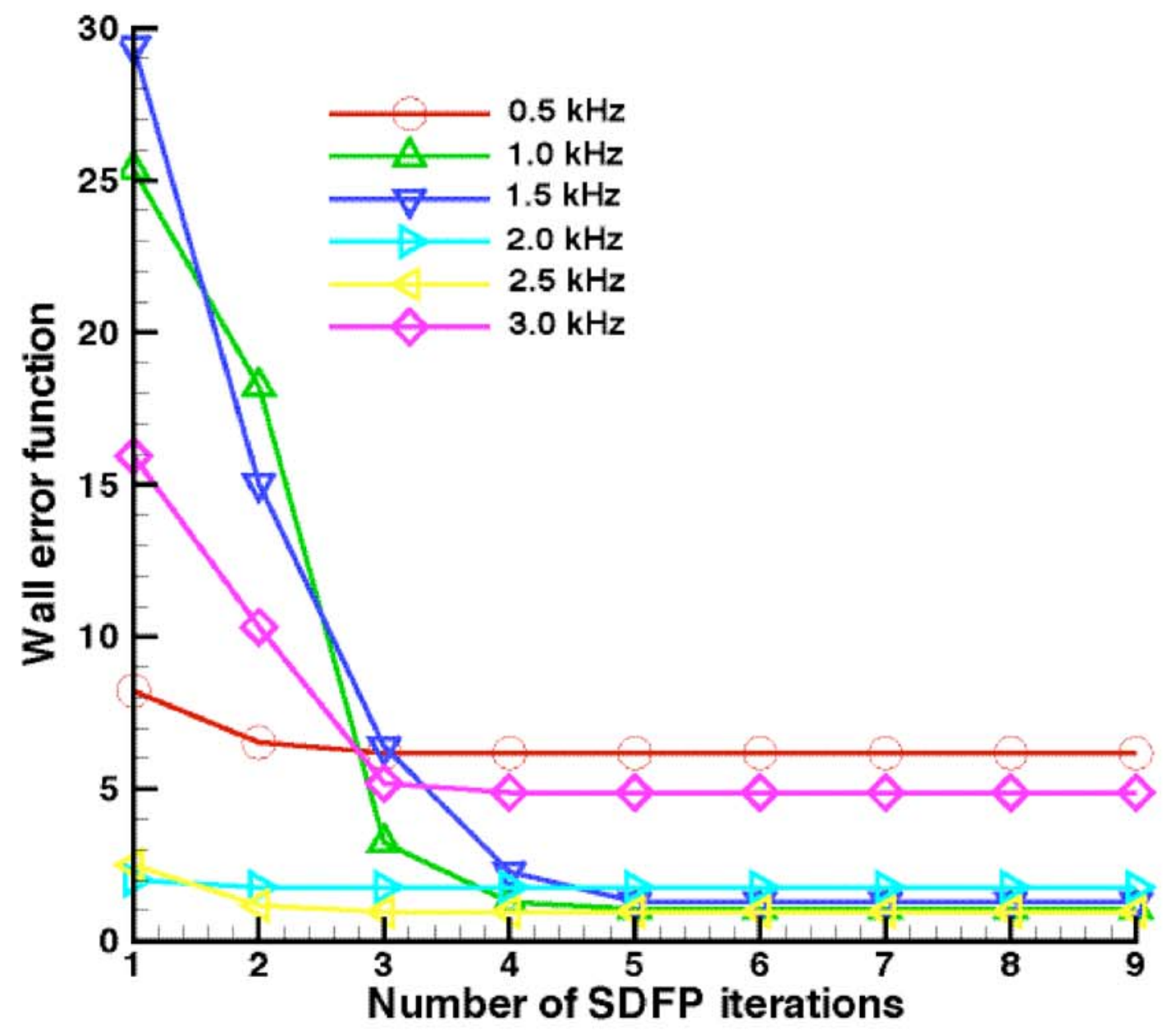

Figure 3. Performance of SDFP algorithm for CT57 test liner on baseline grid $\left(M_{0}=0.255\right)$. 


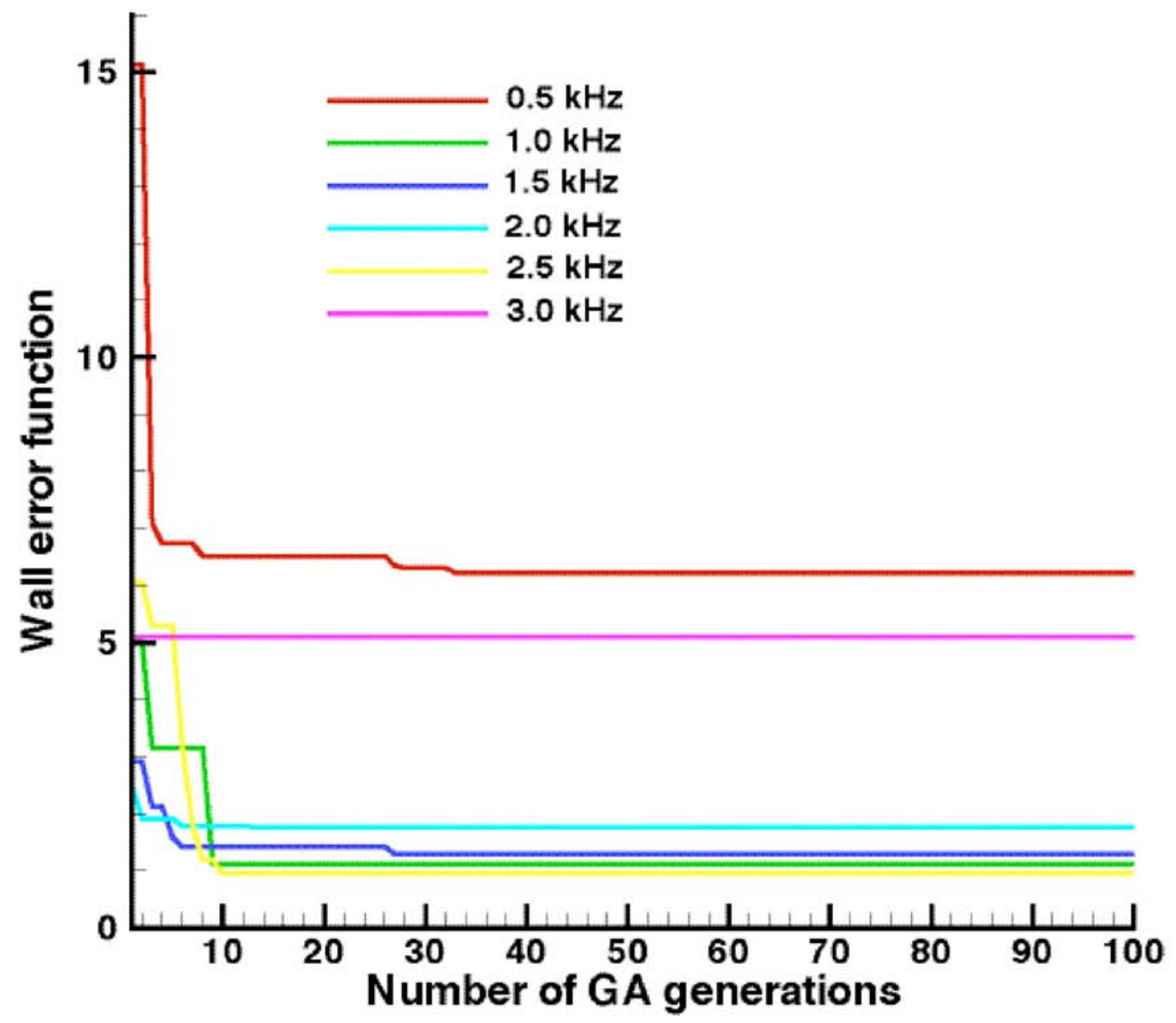

Figure 4. Performance of GA algorithm for CT57 test liner on baseline grid $\left(M_{0}=0.255\right)$. 


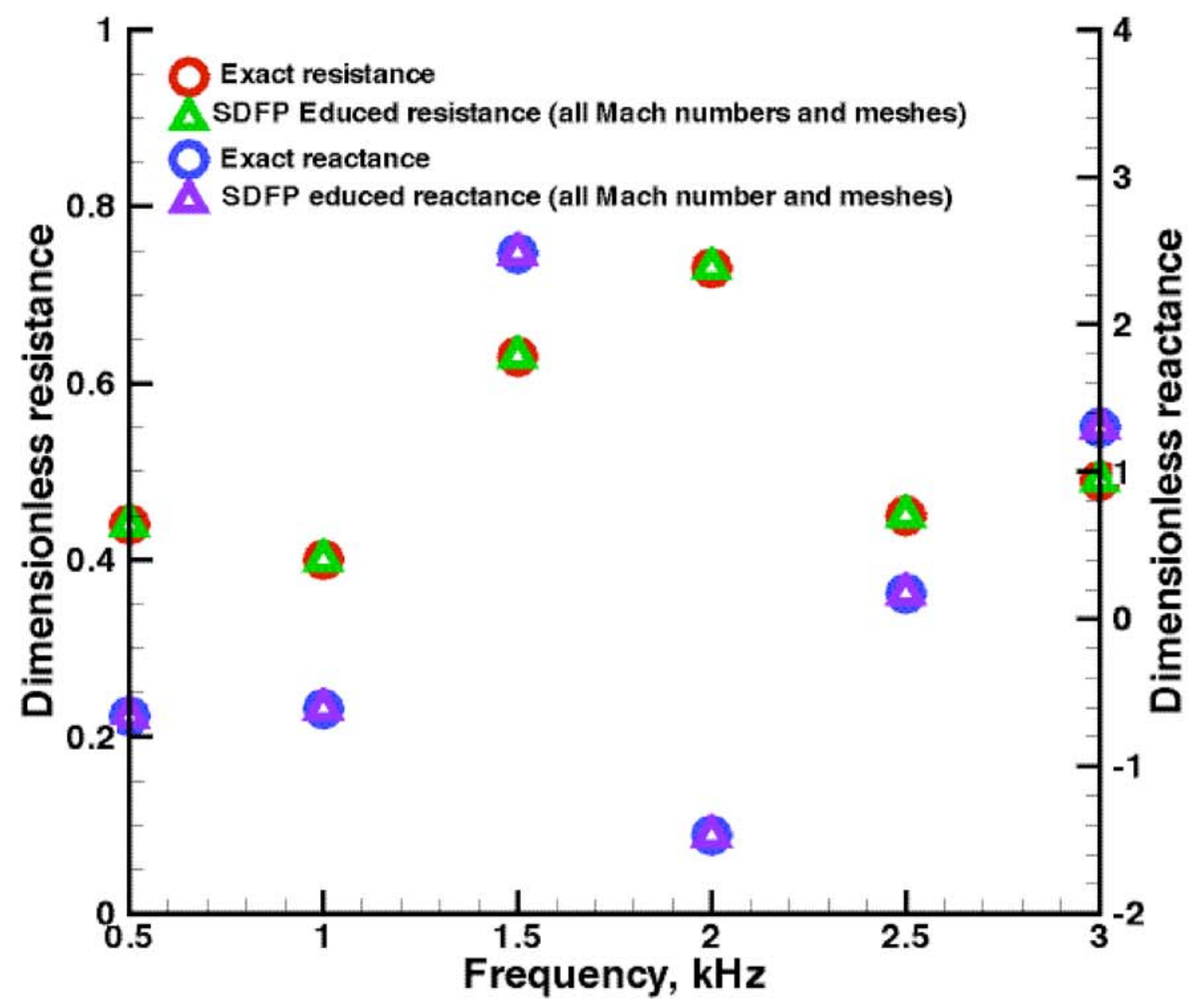

Figure 5. SDFP educed impedance from the Convected Helmholtz model for the CT65 liner. 


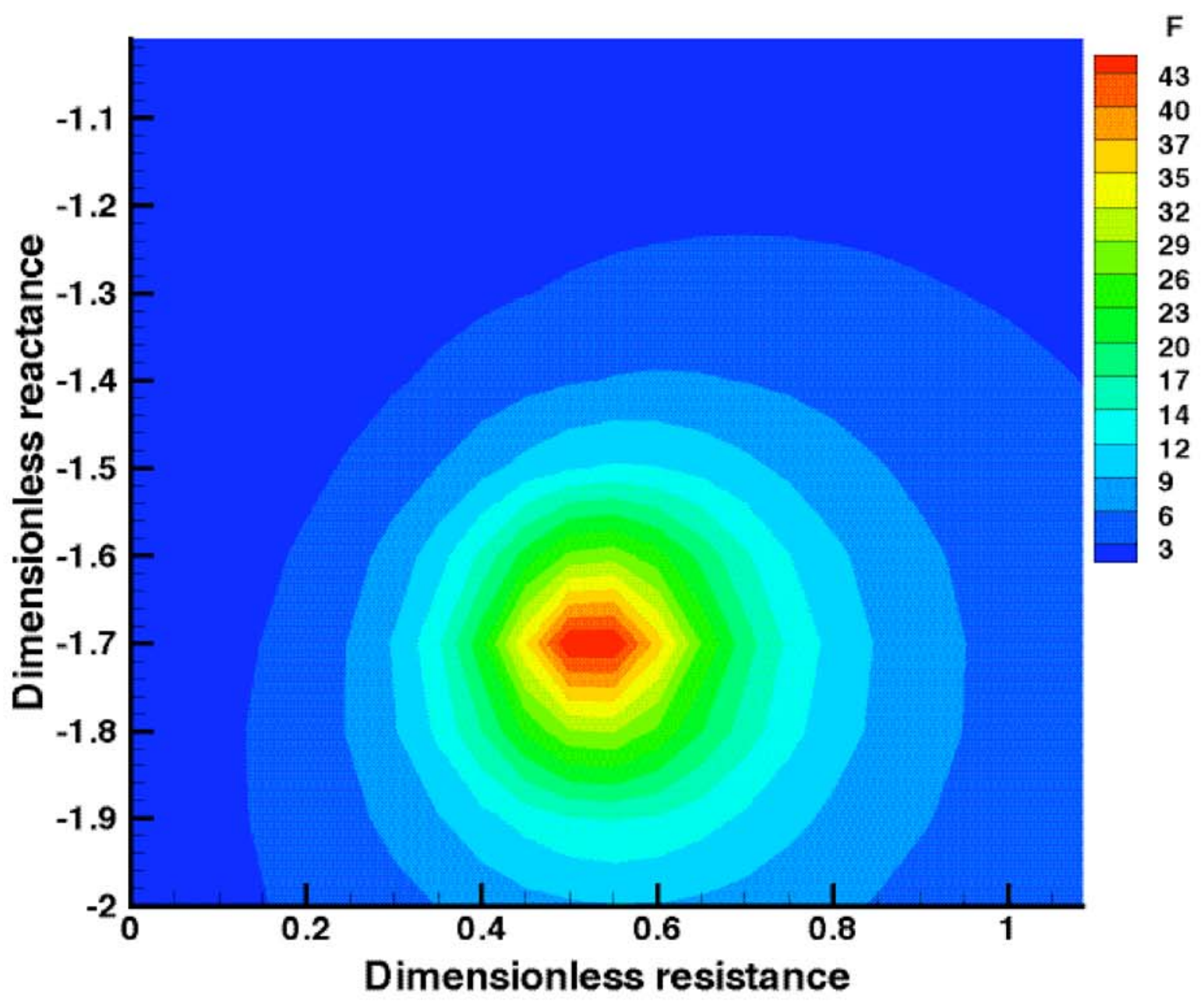

Figure 6. Contour Map of the wall error function at $0.5 \mathrm{kHz}$ for the CT57 liner $\left(M_{0}=0.0\right)$. 


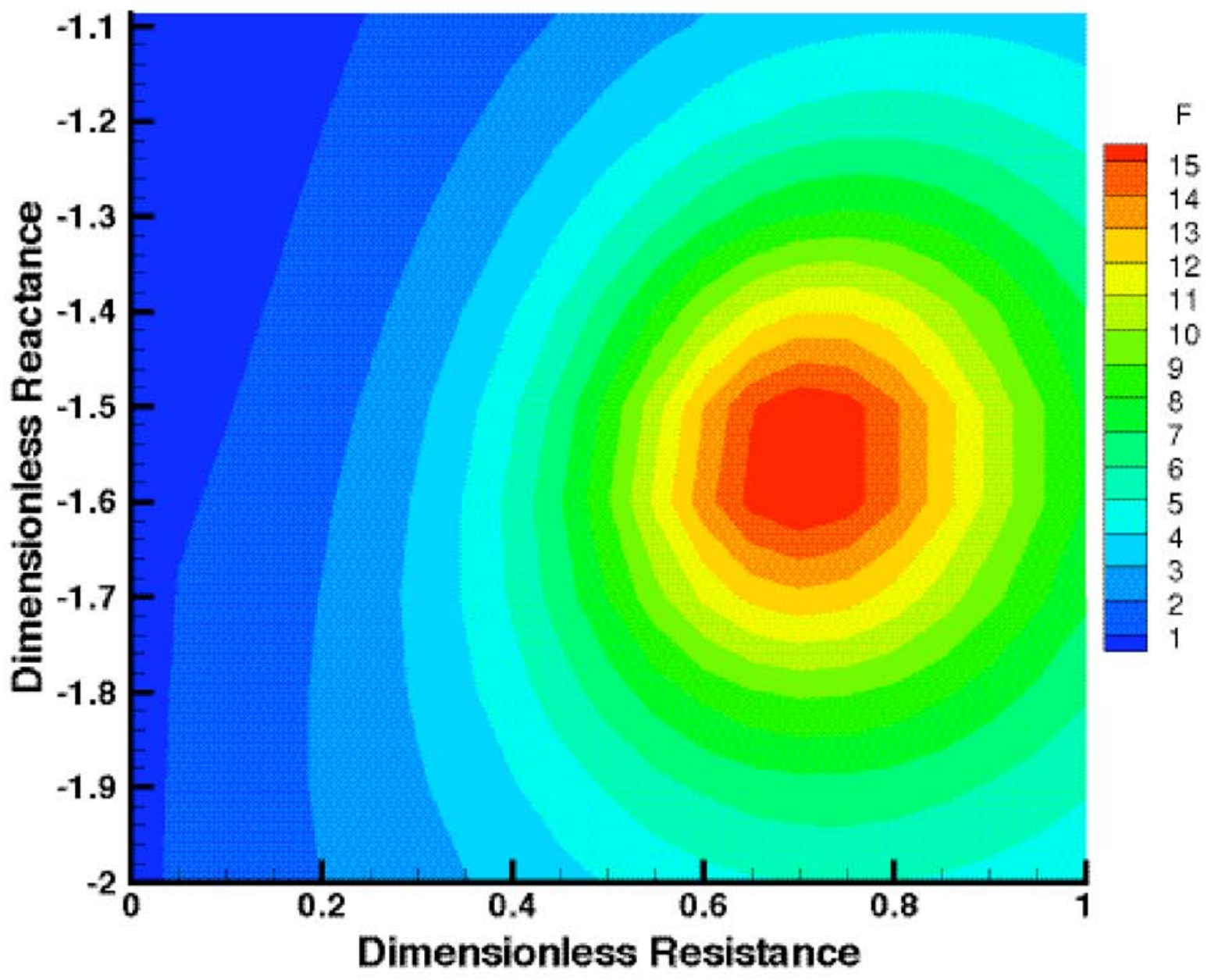

Figure 7. Contour Map of the wall error function at $0.5 \mathrm{kHz}$ for the CT57 liner $\left(M_{0}=0.079\right)$. 


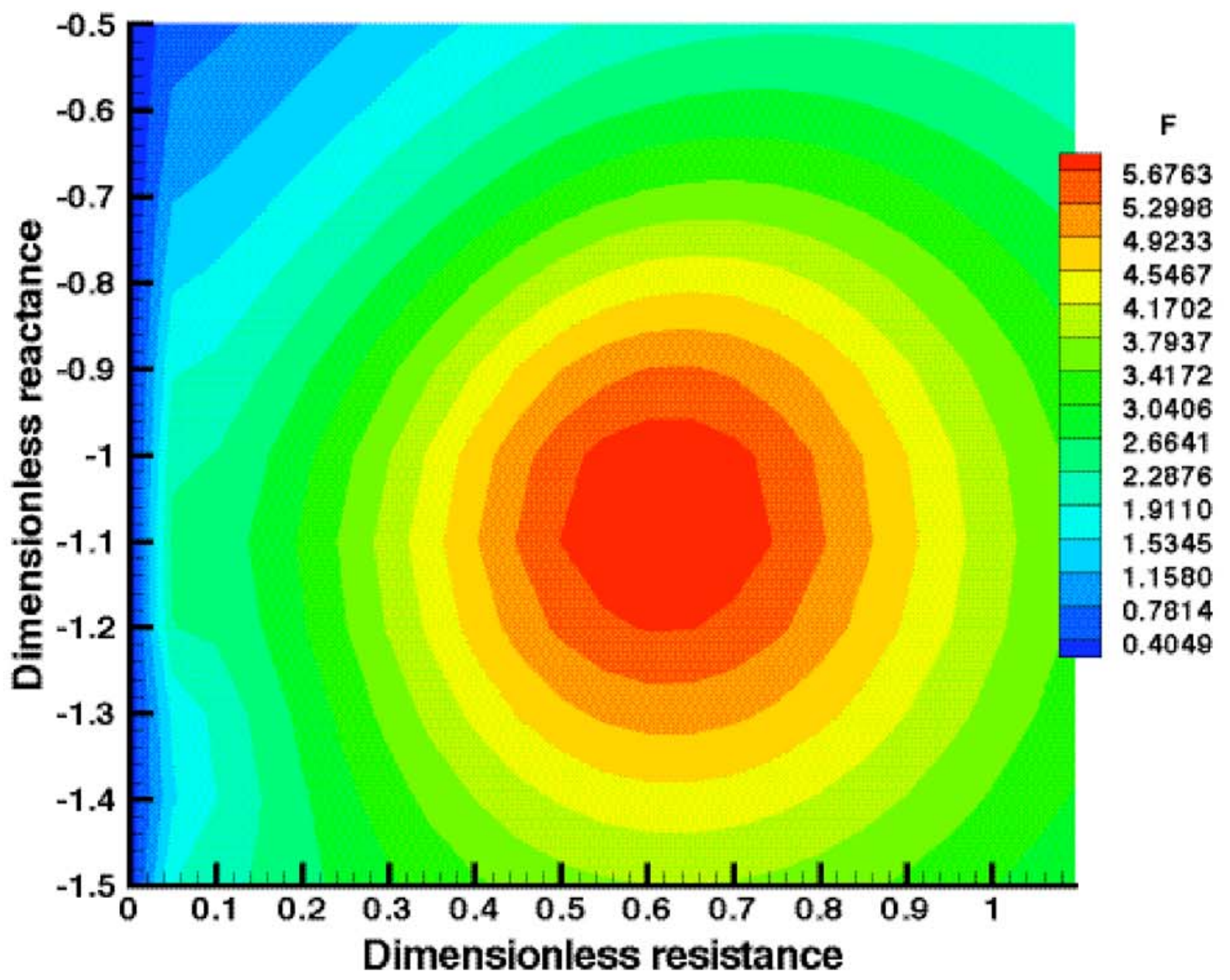

Figure 8. Contour Map of the wall error function at $0.5 \mathrm{kHz}$ for the CT57 liner $\left(M_{0}=0.255\right)$. 


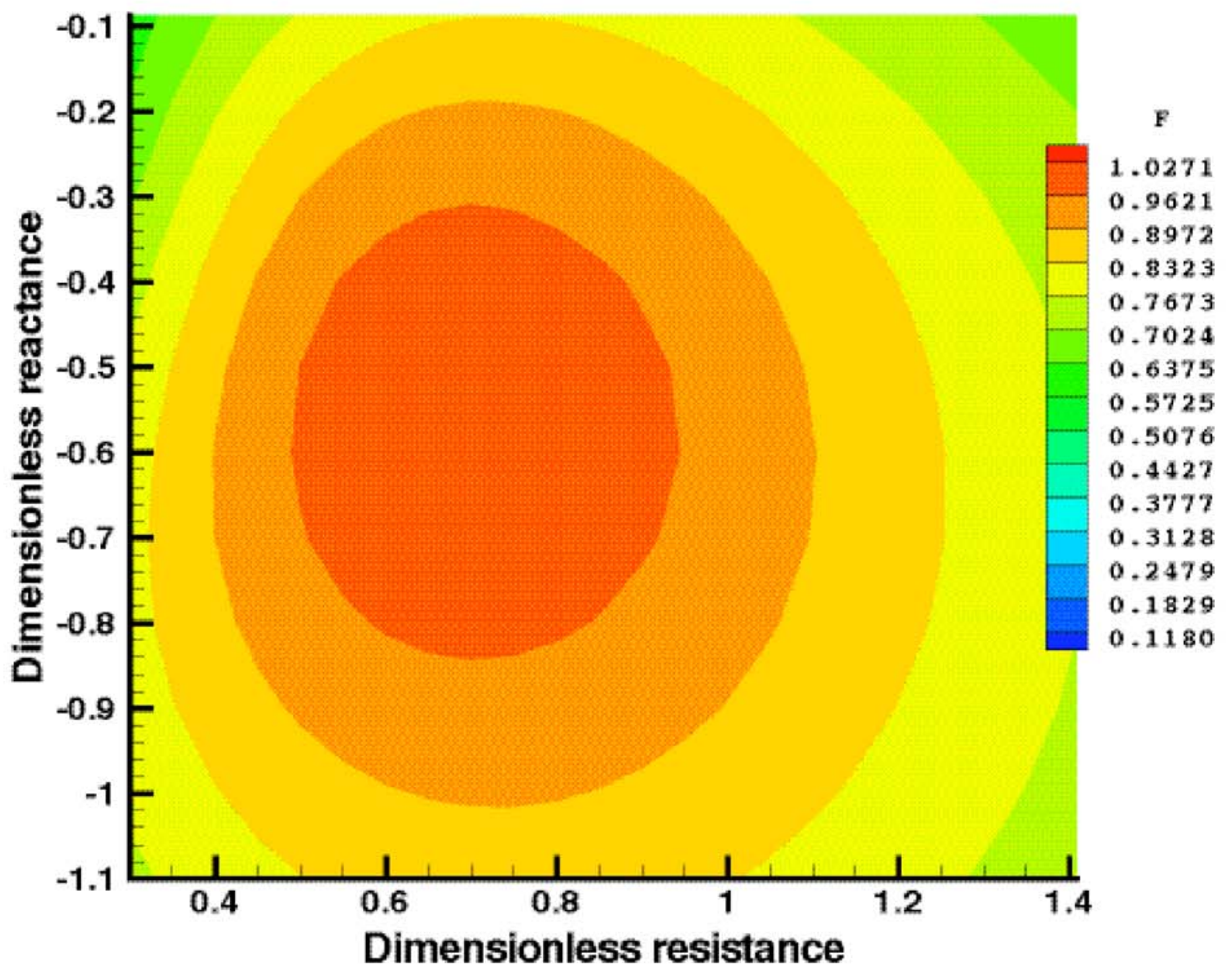

Figure 9. Contour Map of the wall error function at $0.5 \mathrm{kHz}$ for the CT57 liner $\left(M_{0}=0.400\right)$. 